\title{
Stable High Order Finite Difference Methods for Wave Propagation and Flow Problems on Deforming Domains
}

\author{
Samira Nikkar
}

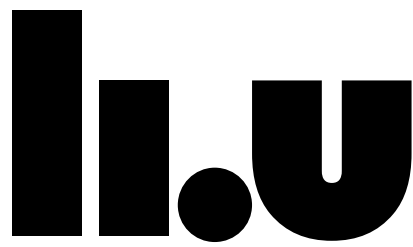

LINKÖPINGS UNIVERSITET

Division of Computational Mathematics

Department of Mathematics

Linköping University

SE-581 83 Linköping, Sweden

Linköping 2016 
Linköping studies in science and technology. Dissertations, No. 1774

Stable High Order Finite Difference Methods for Wave Propagation and Flow Problems on Deforming Domains Samira Nikkar

ISBN 978-91-7685-737-3

ISSN 0345-7524

(C) 2016 Samira Nikkar, unless otherwise noted. All rights reserved.

Samira Nikkar

samira.nikkar@liu.se

www. Iiu.se

Division of Computational Mathematics

Department of Mathematics

Linköping University

SE-581 83 Linköping

Sweden

The cover: The figures on the cover show a pressure pulse governed by the linearized Euler equations coupled along a moving interface with the techniques introduced in paper III.

Typeset with $\mathrm{LAT}_{\mathrm{E}} \mathrm{X} 2 \varepsilon$

Printed by LiU-Tryck, Linköping, Sweden 2016 
To Vaheed and my family 



\section{Abstract}

We construct stable, accurate and efficient numerical schemes for wave propagation and flow problems posed on spatial geometries that are moving, deforming, erroneously described or non-simply connected. The schemes are on Summation-by-Parts (SBP) form, combined with the Simultaneous Approximation Term (SAT) technique for imposing initial and boundary conditions. The main analytical tool is the energy method, by which well-posedness, stability and conservation are investigated. To handle the deforming domains, time-dependent coordinate transformations are used to map the problem to fixed geometries.

The discretization is performed in such a way that the Numerical Geometric Conservation Law (NGCL) is satisfied. Additionally, even though the schemes are constructed on fixed domains, time-dependent penalty formulations are necessary, due to the originally moving boundaries. We show how to satisfy the NGCL and present an automatic formulation for the penalty operators, such that the correct number of boundary conditions are imposed, when and where required.

For problems posed on erroneously described geometries, we investigate how the accuracy of the solution is affected. It is shown that the inaccurate geometry descriptions may lead to wrong wave speeds, a misplacement of the boundary condition, the wrong boundary operator or a mismatch of data. Next, the SBP-SAT technique is extended to time-dependent coupling procedures for deforming interfaces in hyperbolic problems. We prove conservation and stability and show how to formulate the penalty operators such that the coupling procedure is automatically adjusted to the variations of the interface location while the NGCL is preserved.

Moreover, dual consistent SBP-SAT schemes for the linearized incompressible Navier-Stokes equations posed on deforming domains are investigated. To simplify the derivations of the dual problem and incorporate the motions of the boundaries, the second order formulation is reduced to first order and the problem is transformed to a fixed domain. We prove energy stability and dual consistency. It is shown that the solution as well as the divergence of the solution converge with the design order of accuracy, and that functionals of the solution are superconverging.

Finally, initial boundary value problems posed on non-simply connected spatial domains are investigated. The new formulation increases the accuracy of the scheme by minimizing the use of multi-block couplings. In order to show stability, the spectrum of the semi-discrete SBP-SAT formulation is studied. We show that the eigenvalues have the correct sign, which implies stability, in combination with the SBP-SAT technique in time. 



\section{Populärvetenskaplig sammanfattning}

Vi studerar stabila, noggranna och effektiva numeriska metoder för vågutbredning och flödesproblem på rörliga, deformerande eller felaktigt beskrivna geometrier. Våra numeriska metoder bygger på Summation-by-Parts (SBP) kombinerat med en svag initial- och randbehandling kallad Simultaneous Approximation Term (SAT). Det främsta analysverktyget är energimetoden, genom vilken välställdhet, stabilitet och konservation undersöks.

Först betraktar vi hyperboliska problem på rörliga och deformerande domäner. Med lämpliga tidsberoende koordinattransformationer kan problemet lösas på en fast geometri, vilket gör problemet lättare att analysera och implementera. Diskretiseringen måste utföras på ett sådant sätt att "the Numerical Geometric Conservation Law" (NGCL) är uppfylld. Dessutom behövs tidsberoende formuleringar på grund av de ursprungligen rörliga ränderna. Vi visar hur man uppfyller NGCL och presenterar en svag formulering så att rätt antal randvillkor automatiskt införs, där de behövs vid varje given tidpunkt.

För problem med felaktigt beskrivna geometrier undersöker vi hur noggrannheten i lösningen påverkas. Vi visar att de felaktiga geometribeskrivningarna kan leda till felaktiga våghastigheter, felaktiga randvillkor eller till felaktiga data. SBP-SAT-tekniken utvidgas till tidsberoende kopplingar av hyperboliska problem. Vi bevisar konservation och stabilitet och hur man formulerar den svaga kopplingen så att den automatiskt anpassas till variationer av gränsytorna och uppfyller NGCL.

Dualkonsistenta SBP-SAT-system för de inkompressibla Navier-Stokes ekvationer på rörliga domäner konstrueras. För att förenkla härledningen av det duala problemet skrivs formuleringen på första ordningens form. Vi bevisar därefter stabilitet och dualkonsistens. Vi visar också att lösningen samt divergensen av lösningen konvergerar med rätt hastighet och att funktionaler av lösningen superkonvergerar. Slutligen löser vi problem på geometrier som inte är enkelt sammanhängande. Den nya formuleringen är stabil och ökar noggrannheten genom att minimera antal gränsytekopplingar. 



\section{Acknowledgment}

Before anything, I would like to express my deepest gratitudes to my advisor, Prof. Jan Nordström who kept me away from losing my way since my first PhD day. Jan, thank you for sharing your expertise with me and teaching me how to coordinate my thoughts as an engineer and a numerical analyst. The work was difficult, but with your tireless helps and supports challenges became easier to overcome. Your supervision in structuring articles and proofreading this thesis was far beyond the expectations from an advisor.

To my fellow MAI colleagues, old and new; thank you all. Every single one of you has been influential in some aspects of my $\mathrm{PhD}$ life, in one way or another. I would like to specifically single out some names: Jolanta, Yixin, Anna, Leslie, Alexandra, Sonja, Spartak, Arpan and David. You guys made my work environment more friendly and joyous. In our research group, I should thank all $\mathrm{PhD}$ students for sharing their research with me and proofreading my articles. Tomas and Hannes are acknowledged for proofreading parts of this thesis. I specifically thank Markus, for being a nice room-mate and a committed Swedish language mentor who kept his promise for more that three years and did not use a single English word when talking to me. During my $\mathrm{PhD}$ years, I was also supported by administrative staff at MAI whom I truly thank, and would like to express specific gratitudes to Madelaine Engström and Monika Gustafsson. I also acknowledge Fredrik Berntsson, for making my questions feel welcomed with an open door right in front of my office, any time I needed.

To my only sister, Sepideh, who made my last year at LiU unbelievably fantastic by her presence among many other supports. To my parents, I know that I cannot properly express my gratitude with words but I will give it a try anyways. Thanks for all your cares, supports and encouragements. Thank you for being there for me unconditionally, through all pretty and less pretty times when I needed you the most.

The last acknowledgment but certainly the most special one belongs to my love and best friend, Vaheed. There is no formulation that can put a bound on our love. Thank you for being you and as Shamloo says in Persian, Man baharam, to zamin. Man zaminam, to derakht. Man derakhtam, to bahar.

Linköping, August 2016 



\section{List of articles}

This thesis is based on the following papers, which are referred to in the text by their roman numerals.

I) S. Nikkar and J. Nordström, Fully discrete energy stable high order finite difference methods for hyperbolic problems in deforming domains, Journal of Computational Physics, 291: 82-98 (2015).

II) J. Nordström and S. Nikkar, Hyperbolic systems of equations posed on erroneous curved domains, Journal of Computational Physics, 308: 438-442 (2016).

III) S. Nikkar and J. Nordström, A fully discrete, stable and conservative summationby-parts formulation for deforming interfaces, submitted (2016).

IV) S. Nikkar and J. Nordström, A dual consistent summation-by-parts formulation for the linearized incompressible Navier-Stokes equations posed on deforming domains, submitted (2016).

V) S. Nikkar and J. Nordström, Summation-by-parts operators for non-simply connected domains, submitted (2016).

\section{Contributions:}

The theoretical analysis have been developed in close collaboration between the authors. The numerical results have been produced by the author of this thesis. The manuscripts have been written by the author of this thesis with the exception of paper $I I$ which was written jointly together with the first author (although a great generosity in editing and structuring has been given to all the manuscripts by my advisor Prof. Jan Nordström). 

Contents

1 Introduction $\quad 1$

2 Well-posedness and stability 3

3 SBP-SAT schemes for fixed domains 5

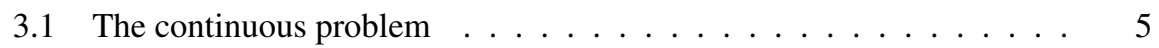

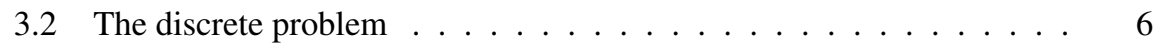

3.3 The interface procedure ............... 8

4 SBP-SAT schemes for deforming domains 11

4.1 The continuous problem ........................ 11

4.2 The discrete problem ...................... 13

4.3 The interface procedure .................. 14

5 Summary of papers $\quad 21$

$\begin{array}{ll}\text { Papers } & 25\end{array}$

I Fully discrete energy stable high order finite difference methods for hyper$\begin{array}{ll}\text { bolic problems in deforming domains } 27 & 27\end{array}$

1 Introduction . . . . . . . . . . . . . . . . . . 28

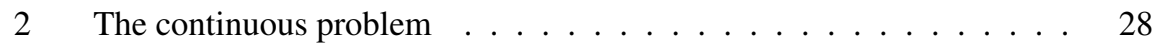

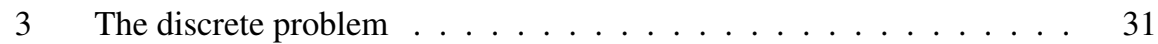

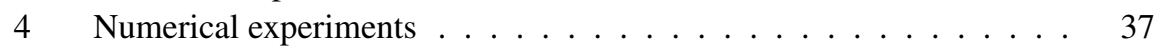

5 Summary and conclusions ................. 44 
II Hyperbolic systems of equations posed on erroneous curved domains

1 Erroneous computational domain . . . . . . . . . . . . 52

2 Numerical experiments . . . . . . . . . . . . . . . . . 54

3 Summary and conclusions ................ 56

III A fully discrete, stable and conservative summation-by-parts formulation for deforming interfaces

1 Introduction . . . . . . . . . . . . . . . . 60

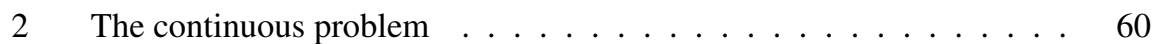

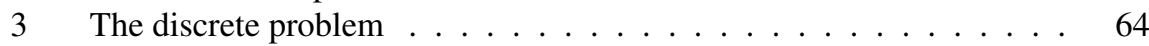

$4 \quad$ Numerical experiments . . . . . . . . . . . . . . . . . . . . . . . . . . 69

5 Summary and conclusions . . . . . . . . . . . . . . . . 80

6 Appendices ......................... 84

IV A dual consistent summation-by-parts formulation for the linearized incompressible Navier-Stokes equations posed on deforming domains 91

1 Introduction . . . . . . . . . . . . . . . . . . 92

2 The incompressible Navier-Stokes equations . . . . . . . . . . . . . 92

3 The discrete problem . . . . . . . . . . . . . . . . . . . 98

4 Numerical experiments . . . . . . . . . . . . . . . . . . . . 106

5 Summary and conclusions . . . . . . . . . . . . . . . 110

V Summation-by-parts operators for non-simply connected domains 113

1 Introduction . . . . . . . . . . . . . . . . . . . . 114

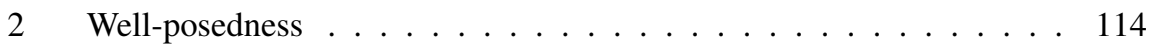

3 Summation-by-part operators . . . . . . . . . . . . . 115

4 Stability ............................ 117

5 Extension to geometries with multiple holes . . . . . . . . . . . . . . 124

6 Numerical experiments . . . . . . . . . . . . . . . . . . . . . . 129

7 Summary and conclusions . . . . . . . . . . . . . . 133

8 Appendices ............................. 135 


\section{Introduction}

Many phenomena in nature can be described by Initial Boundary Value Problems (IBVPs). Examples include the Maxwell equations in electromagnetics, the elastic wave equation in solid mechanics and the Euler or Navier-Stokes equations in fluid dynamics. An analytical solution to an IBVP is, in most cases, impossible to obtain. Therefore, the only choice is to compute an approximation to the solution by discretizing the governing equation(s), using numerical methods and letting computers do the arithmetics. This procedure, together with recent developments in computer science and technology, has made numerical simulations a powerful tool in the analysis of natural phenomena.

In scientific computing, it is important to design stable, accurate and efficient numerical simulations, once a well-posed IBVP is available. Stability deals with nonphysical growth of the solution and accuracy measures how the numerical solution deviates from the exact one. A numerical method is said to be of order $p$, when the error $e$ behaves as $e=\mathcal{O}\left(h^{p}\right)$ where $h$ is a typical mesh size and $p$ is a natural number. Methods with $p>2$ are considered high order accurate. High order methods are computationally more involved by performing more arithmetics per grid point, compared to low order methods. Nevertheless, from an efficiency point of view, stable high order methods are more rapidly converging and give desired error tolerances on coarser grids [1].

In this thesis, we approximate IBVPs by using finite difference formulations on Summation-by-Parts (SBP) form [2, 3] with a weak imposition of initial and boundary conditions. SBP operators are useful since they mimic the integration-by-part property of the continuous derivatives. The weak implementation of initial and boundary conditions is done by using a technique called the Simultaneous Approximation Term (SAT) [7]. The combined use of SBP operators and SAT terms, referred to as the SBP-SAT technique, yields stable, accurate and efficient numerical schemes.

The SBP-SAT technique has so far dealt mostly with IBVPs posed on steady and simply connected spatial domains $[8,9,10,11,13,14,12]$ while many real world 
applications involve more complicated geometries. In this thesis, the SBP-SAT technique is extended to IBVPs posed on moving and deforming spatial domains. In this general context we have investigated the effects of erroneous geometry descriptions on the accuracy of the solution. Further, SBP-SAT schemes for multi-domain coupling procedures where the interface is moving and deforming are formulated and analyzed. Additionally, dual consistent schemes on SBP-SAT form for the linearized incompressible Navier-Stokes equations posed on time-dependent spatial domains are constructed. Finally, SBP-SAT schemes that accurately handle non-simply connected features of the spatial geometry are studied. 


\section{Well-posedness and stability}

Prior to constructing numerical schemes, a study of well-posedness is essential. If the IBVP is not well-posed, small errors in data can be magnified and destroy the accuracy of the solution even without the additional complications related to the discrete scheme. The original definition of well-posedness is given by Hadamard [4] which roughly speaking states that an IBVP is well-posed if

1. a solution exists,

2. the solution is unique,

3. the solution depends smoothly on the data.

The first two statements are intuitively well defined. In contrast, it is not immediately clear how the third requirement should be formulated.

Consider an IBVP of the form

$$
\begin{array}{rlrlrl}
\frac{d}{d t} u & =P(u)+F, & & x \in \Omega, & & t \geq 0, \\
B u & =g, & & x \in \delta \Omega, & t \geq 0, \\
u & =f, & & x \in \Omega, & & t=0,
\end{array}
$$

where $u$ is the solution, $P$ is the differential operator, $B$ is the boundary operator and $\Omega$ is the spatial domain with boundary $\delta \Omega$. Additionally, $F$ is the forcing function, $g$ is the boundary data and $f$ is the initial data. The problem $(2.1)$ is well-posed $[5,15]$ if a unique solution exists and the solution satisfies an energy estimate of the type

$$
\|u\|_{I}^{2} \leq K\left(\|f\|_{I I}^{2}+\|g\|_{I I I}^{2}+\|F\|_{I V}^{2}\right),
$$

where $K$ is independent of $f, g$ and $F$. The norms $\|\cdot\|_{I}$ and $\|\cdot\|_{I I}$ in (2.2) are in general equal while $\|\cdot\|_{I I I}$ and $\|\cdot\|_{I V}$ differ from the others. In this thesis we derive estimates similar to (2.2), by using the energy method as the main analytical tool. 
The counterpart of well-posedness for the continuous problem is the concept of stability for the discrete problem. Similar to the continuous case, stability for a numerical scheme is proven by showing that the numerical solution satisfies an estimate similar to (2.2), and this is most often done by using the discrete version of the energy method. In the next two chapters we will schematically describe how to obtain estimates like (2.2) for a model problem posed on fixed and deforming domains. 


\section{SBP-SAT schemes for fixed domains}

In this section, the standard SBP-SAT procedure is reviewed for fully discrete approximations of IBVPs posed on fixed domains.

\subsection{The continuous problem}

Let the constant coefficient advection equation,

$$
\begin{aligned}
u_{t} & =-a u_{x}, \quad x \in[0,1], \quad t \in[0, T], \\
u(0, x) & =f(x), \quad x \in[0,1], \\
u(t, 0) & =g(t), \quad t \in[0, T],
\end{aligned}
$$

serve as our model IBVP, where $u(t, x)$ is the solution and subscripts $t$ and $x$ denote partial derivatives with respect to time and space. In (3.1), $a>0, f$ is the initial data and $g$ is the boundary data. For simplicity and without restriction we neglect the interference of a forcing function.

By applying the energy method (multiplying the PDE with the solution and integrating in time and space), we find

$$
\|u(T, x)\|^{2}+a \int_{0}^{T} u(t, 1)^{2} d t=a \int_{0}^{T} g(t)^{2} d t+\|f(x)\|^{2} .
$$

In (3.2), the norm is defined by the standard $L_{2}$ inner product and the solution is estimated in terms of data. Uniqueness follows directly from (3.2) together with the linearity of the problem (by considering an identical problem with perturbed data). Existence is due to the use of one boundary condition at $x=0$.

In the presence of a diffusive term, the PDE in (3.1) becomes

$$
u_{t}=-a u_{x}+\varepsilon u_{x x}
$$


where $\varepsilon>0$. A transformation of the kind $w=u_{x}$ takes (3.3) to a first order system,

$$
S U_{t}=B U_{x}+C U
$$

where $U=[u, w]^{T}$ and

$$
S=\left[\begin{array}{ll}
1 & 0 \\
0 & 0
\end{array}\right], B=\left[\begin{array}{cc}
-a & \varepsilon \\
\varepsilon & 0
\end{array}\right], C=\left[\begin{array}{cc}
0 & 0 \\
0 & -\varepsilon
\end{array}\right]
$$

Well-posedness of (3.3) is studied by applying the energy method to (3.4) where we treat the problem as a singular hyperbolic system with a source term. It can be shown that the system (3.4) requires two boundary conditions for well-posedness.

Remark 1. The reduction of (3.3) to (3.4) is used in paper IV where the linearized incompressible Navier-Stokes equations are reduced to a first order system.

\subsection{The discrete problem}

We discretize by constructing an equidistant spatial grid of size $N+1$ arranged as $\mathbf{x}=\left[x_{0}, x_{1}, \ldots, x_{N}\right]^{T}$, and in time we introduce $K+1$ uniform steps. The numerical solution is arranged as

$$
v=\left[\begin{array}{c}
v_{0} \\
\vdots \\
{[v]_{k}} \\
\vdots \\
v_{K}
\end{array}\right],[v]_{k}=\left[\begin{array}{c}
v_{0} \\
\vdots \\
v_{j} \\
\vdots \\
v_{N}
\end{array}\right]_{k}
$$

where $v_{j k} \approx u\left(t_{k}, x_{j}\right)$. In Figure 3.1, a schematic of the fully discrete grid and the indexing used in (3.6) are shown.

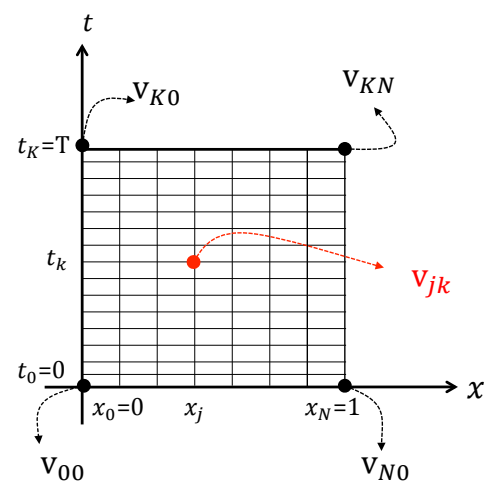

Figure 3.1: A schematic of the fully discrete grid and the indexing used in the arrangement of the numerical solution. 
A first derivative SBP operator of order $s$ is a matrix, $D \in R^{(N+1) \times(N+1)}$, that approximates $\partial / \partial x$ exactly as

$$
\begin{aligned}
& D \mathbf{x}^{i}=P^{-1} Q \mathbf{x}^{i}=i \mathbf{x}^{i-1}, i \in\{0,1, \ldots, s\}, \\
& P=P^{T}>0, \\
& Q+Q^{T}=E_{N}-E_{0},
\end{aligned}
$$

where $E_{0}=\operatorname{diag}(1,0, \ldots, 0)$ and $E_{N}=\operatorname{diag}(0, \ldots, 0,1)$. The matrix $P$ can either be diagonal or block-diagonal and defines the following norm

$$
\|v\|_{P}^{2}=v^{T} P v .
$$

The $P$ norm in (3.8) is a discrete approximation of the standard $L_{2}$ norm. The almost skew-symmetric nature of $Q$ and the discrete norm $P$ lead to the SBP property

$$
\phi^{T} P(D \psi)=\phi^{T} Q \psi=\left(\phi_{N} \psi_{N}-\phi_{0} \psi_{0}\right)-(D \phi)^{T} P \psi
$$

for discrete grid functions $\phi$ and $\psi$.

First derivative SBP operators with interior accuracy of order $2 s$ for $s \in\{1,2,3,4,5\}$ and boundary closures of $s$ order accurate are available, see for example [3,6]. Together with a diagonal matrix $P$, the global order of accuracy becomes $s+1[3,17,18]$ for hyperbolic pointwise stable schemes. For comprehensive reviews of the SBP-SAT schemes, see $[15,16]$.

One-dimensional SBP operators in $x$ and $t$ (for SBP techniques in time see [20, 21, $22]$ ), are extended to the fully discrete grid, as

$$
\mathcal{D}_{t}=D_{t} \otimes I_{x}, \mathcal{D}_{x}=I_{t} \otimes D_{x},
$$

where $\otimes$ denotes the Kronecker product. In (3.10), the matrices in the first and second Kronecker positions are of size $K+1$ and $N+1$, respectively. Additionally, $I$ is the identity matrix with a relevant size.

The fully discrete SBP-SAT version of (3.1) is

$$
\mathcal{D}_{t} v=-a \mathcal{D}_{x} v+\sigma_{t}\left(P_{t}^{-1} E_{0} \otimes I_{x}\right)(v-\mathbf{f})+\sigma_{L}\left(I_{t} \otimes P_{x}^{-1} E_{0}\right)(v-\mathbf{g}),
$$

where $\sigma_{t}$ and $\sigma_{L}$ are penalty parameters for the weak initial and boundary conditions that will be derived based on stability requirements. In $(3.11), E_{0}=\operatorname{diag}(1, \ldots, 0)$ has a size consistent with its Kronecker position, $\mathbf{f}$ and $\mathbf{g}$ are vectors with initial and boundary data injected in relevant positions.

The stability is investigated via the discrete version of the energy method, i.e. by multiplying (3.11) from the left by $v^{T} \mathcal{P}$ where $\mathcal{P}=\left(P_{t} \otimes P_{x}\right)$. Adding the transpose of the result to itself and completing the squares give

$$
\begin{aligned}
v^{T}\left(E_{K} \otimes P_{x}\right) v & =\left(a+2 \sigma_{L}\right)\left(v-\frac{\sigma_{L}}{a+2 \sigma_{L}} \mathbf{g}\right)\left(P_{t} \otimes E_{0}\right)\left(v-\frac{\sigma_{L}}{a+2 \sigma_{L}} \mathbf{g}\right)-\frac{\sigma_{L}^{2}}{a+2 \sigma_{L}} \mathbf{g}^{T}\left(P_{t} \otimes E_{0}\right) \mathbf{g} \\
& +\left(1+2 \sigma_{t}\right)\left(v-\frac{\sigma_{t}}{1+2 \sigma_{t}} \mathbf{f}\right)\left(E_{0} \otimes P_{x}\right)\left(v-\frac{\sigma_{t}}{1+2 \sigma_{t}} \mathbf{f}\right)-\frac{\sigma_{t}^{2}}{1+2 \sigma_{t}} \mathbf{f}^{T}\left(E_{0} \otimes P_{t}\right) \mathbf{f} \\
& -a v^{T}\left(P_{t} \otimes E_{N}\right) v,
\end{aligned}
$$


where $E_{K, N}=\operatorname{diag}(0, \ldots, 1)$. Clearly (3.12) is stable for $\sigma_{t} \leq-1 / 2$ and $\sigma_{L} \leq-a / 2$. By choosing $\sigma_{t}=-1$ and $\sigma_{L}=-a$, (3.12) takes a particular nice form given by

$$
\begin{aligned}
\left\|v_{K}\right\|_{H_{K}}^{2}+a v^{T}\left(P_{t} \otimes E_{N}\right) v & =a \mathbf{g}^{T}\left(P_{t} \otimes E_{0}\right) \mathbf{g}-a(v-\mathbf{g})^{T}\left(P_{t} \otimes E_{0}\right)(v-\mathbf{g}) \\
& +\|\mathbf{f}\|_{H_{0}}^{2}-\left\|v_{0}-\mathbf{f}\right\|_{H_{0}}^{2},
\end{aligned}
$$

where the subscripts $K$ and 0 restrict the solution to $t_{K}=T$ and $t_{0}=0$. Additionally, $\left\|v_{K, 0}\right\|_{H_{K, 0}}^{2}=v_{K, 0}^{T} H_{K, 0} v_{K, 0}$ where $H_{K, 0}=E_{K, 0} \otimes P_{x}$ are positive definite when acting on the solutions $v_{K, 0}$, respectively. The estimate in (3.13), guarantees stability and is similar to the continuous result in (3.2), but with added damping terms due to the weak implementations.

\subsection{The interface procedure}

In this section, the fully discrete SBP-SAT procedure for a fixed interface procedure is discussed.

\subsubsection{The continuous case}

Consider the constant coefficient advection equation with a solution $u$ posed on two nearby spatial domains, as

$$
\begin{aligned}
& u_{t}^{L}=-a u_{x}^{L}, x \in[-1,0], \\
& u_{t}^{R}=-a u_{x}^{R}, x \in[0,1],
\end{aligned}
$$

where $a>0$. The solutions $u^{L}$ and $u^{R}$ represent, respectively, the left and right values of the continuous solution $u$. In the following, we omit the outer boundary condition and only focus on the interface.

\section{Conservation}

We start by multiplying (3.14) with a smooth function $\phi(t, x)$ with compact support at the temporal and spatial boundaries. Integration in time and space yield

$$
\int_{0}^{T} \int_{-1}^{0} u^{L}\left(\phi_{t}+a \phi_{x}\right) d x d t+\int_{0}^{T} \int_{0}^{1} u^{R}\left(\phi_{t}+a \phi_{x}\right) d x d t=\int_{0}^{T} a \phi\left(u^{L}-u^{R}\right) d t .
$$

From (3.16), it is trivial to see that the continuity condition $u^{L}=u^{R}$ removes the interface term which shows conservation.

\section{Well-posedness}

Applying the energy method to (3.14) lead to

$$
\begin{aligned}
\left\|u^{L}(T, x)\right\|^{2}+\left\|u^{R}(T, x)\right\|^{2}= & -a \int_{0}^{T}\left(u^{L}(t, 0)\right)^{2} d t+a \int_{0}^{T}\left(u^{R}(t, 0)\right)^{2} d t \\
& -\left\|u^{L}(0, x)\right\|^{2}-\left\|u^{R}(0, x)\right\|^{2} .
\end{aligned}
$$

Continuity at the interface together with the initial conditions $u^{L}=f^{L}$ and $u^{R}=f^{R}$ results in

$$
\left\|u^{L}(T, x)\right\|^{2}+\left\|u^{R}(T, x)\right\|^{2}=\left\|f^{L}\right\|^{2}+\left\|f^{R}\right\|^{2} .
$$




\subsubsection{The discrete case}

We construct uniform grids of size $N+1$ in $x \in[-1,0], M+1$ in $x \in[0,1]$ and $K+1$ in time. The fully discrete SBP-SAT version of (3.14) including weak initial and interface conditions becomes

$$
\begin{aligned}
& \mathcal{D}_{t} v^{L}=-a \mathcal{D}_{x}^{L} v^{L}+\sigma_{t}^{L}\left(P_{t}^{-1} E_{0} \otimes I_{x}\right)\left(v^{L}-\mathbf{f}^{L}\right)+\sigma^{L}\left[I_{t} \otimes\left(P_{x}^{L}\right)^{-1} E_{N}\right]\left(v^{L}-\mathbf{v}_{I}^{R}\right), \\
& \mathcal{D}_{t} v^{R}=-a \mathcal{D}_{x}^{R} v^{R}+\sigma_{t}^{R}\left(P_{t}^{-1} E_{0} \otimes I_{x}\right)\left(v^{R}-\mathbf{f}^{R}\right)+\sigma^{R}\left[I_{t} \otimes\left(P_{x}^{R}\right)^{-1} E_{0}\right]\left(v^{R}-\mathbf{v}_{I}^{L}\right),
\end{aligned}
$$

where $\sigma_{t}^{L, R}$ are penalty coefficients for the weak imposition of the left and right initial conditions. Additionally, $\sigma^{L, R}$ are penalty coefficients for the weak imposition of the left and right interface conditions. By $\mathbf{v}_{I}^{R}$ and $\mathbf{v}_{I}^{L}$ we denote vectors containing the solutions of the right and left schemes restricted to the interface. The bold face indicates that the size and arrangement of these vectors are modified to be consistent with the schemes they are operating in. At the interface location both numerical solutions approximate $u(t, 0)$, but are not necessarily equal. In addition, $D_{x}^{L, R}$ may have different orders of accuracy.

\section{Discrete conservation}

The discrete conservation property of the approximation is investigated by multiplying (3.18) with $\phi^{T} \mathcal{P}^{L, R}$ where $\mathcal{P}^{L, R}=P_{t} \otimes P_{x}^{L, R}$. The result is

$$
\begin{array}{r}
{\left[\left(v^{L}\right)^{T} \mathcal{P}^{L}\left(\mathcal{D}_{t}+a \mathcal{D}_{x}^{L}\right) \phi\right]^{T}+\left[\left(v^{R}\right)^{T} \mathcal{P}^{R}\left(\mathcal{D}_{t}+a \mathcal{D}_{x}^{R}\right) \phi\right]^{T}=} \\
\phi_{I}^{T} P_{t}\left(a+\sigma^{R}-\sigma^{L}\right)\left(v_{I}^{L}-v_{I}^{R}\right),
\end{array}
$$

where the subscript $I$ indicates interface. The matrices and vectors on the right hand side of (3.19) are now all of size $K+1$. The right hand side of (3.19) vanishes if the conservation condition

$$
a+\sigma^{R}-\sigma^{L}=0
$$

holds.

\section{Stability}

The discrete energy method applied to (3.18) and the choice $\sigma_{t}^{L, R}=-1$ lead to

$$
\begin{aligned}
\left\|v_{K}^{L}\right\|_{H_{K}^{L}}^{2}+\left\|v_{K}^{R}\right\|_{H_{K}^{R}}^{2} & =\left[\begin{array}{c}
v_{I}^{L} \\
v_{I}^{R}
\end{array}\right]^{T}\left(\left[\begin{array}{cc}
2 \sigma^{L}-a & -\left(\sigma^{L}+\sigma^{R}\right) \\
-\left(\sigma^{L}+\sigma^{R}\right) & 2 \sigma^{R}+a
\end{array}\right] \otimes\left[\begin{array}{cc}
P_{t} & 0 \\
0 & P_{t}
\end{array}\right]\right)\left[\begin{array}{c}
v_{I}^{L} \\
v_{I}^{R}
\end{array}\right] \\
& +\left\|\mathbf{f}^{L}\right\|_{H_{0}^{L}}^{2}-\left\|v_{0}^{L}-\mathbf{f}^{L}\right\|_{H_{0}^{L}}^{2}+\left\|\mathbf{f}^{R}\right\|_{H_{0}^{R}}^{2}-\left\|v_{0}^{R}-\mathbf{f}^{R}\right\|_{H_{0}^{R}}^{2} .
\end{aligned}
$$

In (3.21), the subscripts $K$ and 0 restrict the solution to $t_{K}=T$ and $t_{0}=0$. Additionally, $H_{K}^{L, R}=E_{K} \otimes P_{x}^{L, R}$ and $H_{0}^{L, R}=E_{0} \otimes P_{x}^{L, R}$. Recall that we ignore the outer boundary condition.

With the use of (3.20), (3.21) becomes

$$
\begin{aligned}
\left\|v_{K}^{L}\right\|_{H_{K}^{L}}^{2}+\left\|v_{K}^{R}\right\|_{H_{K}^{R}}^{2} & =\left(v_{I}^{L}-v_{I}^{R}\right)^{T} P_{t}\left(-a+2 \sigma^{L}\right)\left(v_{I}^{L}-v_{I}^{R}\right) \\
& +\left\|\mathbf{f}^{L}\right\|_{H_{0}^{L}}^{2}-\left\|v_{0}^{L}-\mathbf{f}^{L}\right\|_{H_{0}^{L}}^{2}+\left\|\mathbf{f}^{R}\right\|_{H_{0}^{R}}^{2}-\left\|v_{0}^{R}-\mathbf{f}^{R}\right\|_{H_{0}^{R}}^{2} .
\end{aligned}
$$


From (3.22), we conclude that

$$
\sigma^{L} \leq a / 2
$$

leads to a stable and conservative interface treatment. We choose $\sigma^{L}=0$ and $\sigma^{R}=-a$ which satisfy (3.20) and (3.23). The energy estimate becomes

$$
\begin{aligned}
\left\|v_{K}^{L}\right\|_{H_{K}^{L}}^{2}+\left\|v_{K}^{R}\right\|_{H_{K}^{R}}^{2}= & -a\left(v_{I}^{L}-v_{I}^{R}\right)^{T} P_{t}\left(v_{I}^{L}-v_{I}^{R}\right) \\
& +\left\|\mathbf{f}^{L}\right\|_{H_{0}^{L}}^{2}-\left\|v_{0}^{L}-\mathbf{f}^{L}\right\|_{H_{0}^{L}}^{2}+\left\|\mathbf{f}^{R}\right\|_{H_{0}^{R}}^{2}-\left\|v_{0}^{R}-\mathbf{f}^{R}\right\|_{H_{0}^{R}}^{2} .
\end{aligned}
$$

which is similar to the continuous energy estimate in (3.17), with extra damping due to the weak imposition of the interface and initial conditions.

The interface procedure is generally used when the spatial domain is complicated from a mesh generation perspective and can be divided into simpler geometries, or in multi-physics applications. One drawback with this procedure is that close to the interface the accuracy of the approximation is reduced from $2 s$ to $s+1$, locally.

Remark 2. In paper $V$ where we study non-simply connected domains, we have increased the accuracy of the standard SBP-SAT formulation, by minimizing the number of interface procedures. 


\section{SBP-SAT schemes for deforming domains}

In this section, the SBP-SAT procedure is extended to fully discrete IBVPs posed on deforming domains, and compared with the results in Section 3.

\subsection{The continuous problem}

Consider the constant coefficient advection equation posed on a deforming domain

$$
u_{t}=-a u_{x}, x \in\left[x_{L}(t), x_{R}(t)\right], t \in[0, T],
$$

where $a>0$. To incorporate the movement of the domain, we introduce an invertible time-dependent Lagrangian-Eulerian coordinate transformation,

$$
x(\tau, \xi) \rightleftharpoons \xi(t, x), \quad t=\tau,
$$

that results in a spatial domain with fixed boundaries. A schematic of such deforming and fixed domains is shown in Figure 4.1.

The transformation satisfies

$$
\left[\begin{array}{l}
\partial / \partial \xi \\
\partial / \partial \tau
\end{array}\right]=\underbrace{\left[\begin{array}{ll}
x_{\xi} & 0 \\
x_{\tau} & 1
\end{array}\right]}_{:=[J]}\left[\begin{array}{l}
\partial / \partial x \\
\partial / \partial t
\end{array}\right],
$$

where the subscripts $\xi$ and $\tau$ denote partial derivatives of the respective direction, and $[J]$ is the Jacobian matrix of transformation. By considering the inverse transformation, the following metric relations are obtained

$$
J \xi_{t}=-x_{\tau}, J \xi_{x}=1
$$



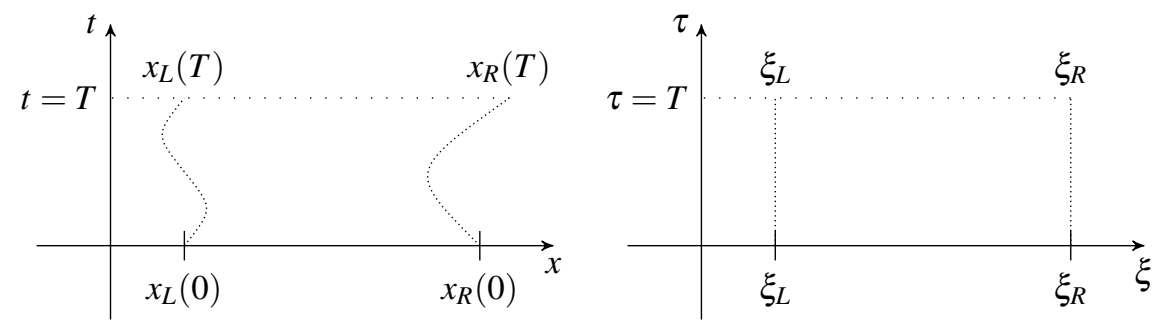

Figure 4.1: A schematic of the deforming and fixed domains.

where $J=x_{\xi}>0$ is the determinant of $[J]$. For all non-singular transformations, the Geometric Conservation Law (GCL) is satisfied, that is

$$
J_{\tau}+\left(J \xi_{t}\right)_{\xi}=0
$$

Next, (4.1) is expressed in terms of $\xi$ and $\tau$ (by using the chain rule), and multiplied with $J$. The result is

$$
J u_{\tau}=-\alpha u_{\xi}, \quad \xi \in\left[\xi_{L}, \xi_{R}\right], \tau \in[0, T],
$$

where $\alpha(\tau, \xi)=J \xi_{t}+a J \xi_{x}$. The energy method applied to (4.6) gives

$$
\int_{0}^{T} \int_{\xi_{L}}^{\xi_{R}}\left(J u^{2}\right)_{\tau} d \xi d \tau=-\int_{0}^{T} \int_{\xi_{L}}^{\xi_{R}}\left(\alpha u^{2}\right)_{\xi} d \xi d \tau+\int_{0}^{T} \int_{\xi_{L}}^{\xi_{R}}\left(\alpha_{\xi}+J_{\tau}\right) u^{2} d \xi d \tau
$$

On the right hand side of (4.7), $\alpha_{\xi}+J_{\tau}=0$ as a result of the GCL (4.5). Hence, (4.7) is equivalent to

$$
\|u(T, \xi)\|_{J}^{2}-\|u(0, \xi)\|_{J}^{2}=\int_{0}^{T} \alpha_{L}(\tau)\left(u_{L}(\tau)\right)^{2} d \tau-\int_{0}^{T} \alpha_{R}(\tau)\left(u_{R}(\tau)\right)^{2} d \tau
$$

where $\|u\|_{J}^{2}=\int_{\xi_{L}}^{\xi_{R}} J u^{2} d \xi, \alpha_{L, R}(\tau)=\alpha\left(\tau, \xi_{L, R}\right)$ and $u_{L, R}(\tau)=u\left(\tau, \xi_{L, R}\right)$.

In (4.8), the energy of the solution is bounded by choosing

$$
\begin{array}{ll}
u_{L}(\tau)=g(\tau) & \text { if } \quad \alpha_{L}>0 \\
u_{R}(\tau)=h(\tau) & \text { if } \quad \alpha_{R}<0
\end{array}
$$

where $g$ and $h$ are given data. Inserting (4.9) into (4.8), and imposing the initial condition $u=f$ lead to the following four possible estimates

$$
\begin{aligned}
& \|u(T, x)\|_{J}^{2}=\|f\|_{J}^{2}+\int_{0}^{T} \alpha_{L}(\tau) g(\tau)^{2} d \tau-\int_{0}^{T} \alpha_{R}(\tau) u_{R}^{2} d \tau, \quad \alpha_{L}>0, \alpha_{R} \geq 0, \\
& \|u(T, x)\|_{J}^{2}=\|f\|_{J}^{2}+\int_{0}^{T} \alpha_{L}(\tau) g(\tau)^{2} d \tau-\int_{0}^{T} \alpha_{R}(\tau) h(\tau)^{2} d \tau, \quad \alpha_{L}>0, \alpha_{R}<0, \\
& \|u(T, x)\|_{J}^{2}=\|f\|_{J}^{2}+\int_{0}^{T} \alpha_{L}(\tau) u_{L}(\tau)^{2} d \tau-\int_{0}^{T} \alpha_{R}(\tau) h(\tau)^{2} d \tau, \quad \alpha_{L} \leq 0, \alpha_{R}<0, \\
& \|u(T, x)\|_{J}^{2}=\|f\|_{J}^{2}+\int_{0}^{T} \alpha_{L}(\tau) u_{L}(\tau)^{2} d \tau-\int_{0}^{T} \alpha_{R}(\tau) u_{R}(\tau)^{2} d \tau, \alpha_{L} \leq 0, \alpha_{R} \geq 0 .
\end{aligned}
$$


Remark 3. The number of well-posed boundary conditions for (4.1) may be zero, one or two, depending on the motion of the boundaries. In the case with fixed boundaries, one boundary condition only leads to well-posedness ( $x=0$ is the inflow boundary for $a>0$ and $x=1$ is the inflow for $a<0)$.

\subsection{The discrete problem}

Prior to constructing the numerical scheme, we apply the splitting technique in [19] required for problems with variable coefficients. This takes (4.6) to

$$
\frac{1}{2}\left[J u_{\tau}+(J u)_{\tau}+J_{\tau} u\right]=-\frac{1}{2}\left[\alpha u_{\xi}+(\alpha u)_{\xi}+\alpha \xi u\right]
$$

where we, for illustrative reasons, keep the factor $1 / 2$ in the scheme.

In $\xi \in\left[\xi_{L}, \xi_{R}\right]$ an equidistant mesh of size $N+1$ and $K+1$ uniform time steps are introduced. The fully discrete SBP-SAT version of (4.11) including weak initial and boundary conditions is

$$
\begin{aligned}
\frac{1}{2}\left[\mathcal{J D}_{\tau} v+\mathcal{D}_{\tau}(\mathcal{J} v)+\mathcal{J}_{\tau} v\right]= & -\frac{1}{2}\left[A \mathcal{D}_{\xi} v+\mathcal{D}_{\xi}(A v)+A \xi v\right] \\
& +\Sigma_{\tau}\left(P_{\tau}^{-1} E_{0} \otimes I_{\xi}\right)(v-\mathbf{f}) \\
& +\Sigma_{L}\left(I_{\tau} \otimes P_{\xi}^{-1} E_{0}\right)(v-\mathbf{g}) \\
& +\Sigma_{R}\left(I_{\tau} \otimes P_{\xi}^{-1} E_{N}\right)(v-\mathbf{h}),
\end{aligned}
$$

where $\mathcal{J}, \mathcal{\partial}_{\tau}, A$ and $A_{\xi}$ are diagonal matrices approximating $J, J_{\tau}, \alpha$ and $\alpha_{\xi}$, pointwise, given by

$$
\begin{aligned}
& \mathcal{J}=\operatorname{diag}\left(\mathcal{D}_{\xi} X\right) \\
& \mathcal{J}_{\tau}=\operatorname{diag}\left[\mathcal{D}_{\tau}\left(\mathcal{D}_{\xi} X\right)\right] \\
& A=a\left(I_{\tau} \otimes I_{\xi}\right)-\operatorname{diag}\left(\mathcal{D}_{\tau} X\right), \\
& A_{\xi}=-\operatorname{diag}\left[\mathcal{D}_{\xi}\left(\mathcal{D}_{\tau} X\right)\right] .
\end{aligned}
$$

In (4.13), $X$ is a vector of size $(K+1) \times(N+1)$ containing $x\left(\tau_{k}, \xi_{j}\right)$ for $k \in\{0,1, \ldots, K\}$ and $j \in\{0,1, \ldots, N\}$, consistent in arrangement to (3.6). Additionally, $\Sigma_{\tau, L, R}$ are diagonal penalty operators for the weak initial, left and right boundary conditions, respectively. Moreover, $E_{0}=\operatorname{diag}(1,0, \ldots, 0)$ and $E_{N}=\operatorname{diag}(0, \ldots, 0,1)$ with relevant sizes depending on their position in the Kronecker product. Finally, $\mathbf{f}, \mathbf{g}, \mathbf{h}$ are vectors with the data $f, g$ and $h$ injected pointwise into the initial time, left and right boundary locations, respectively.

The discrete energy method (multiplying (4.12) with $v^{T} \mathcal{P}$ where $\left.\mathcal{P}=\left(P_{\tau} \otimes P_{\xi}\right)\right)$ and adding the transpose result in

$$
\begin{aligned}
v^{T} \mathcal{J}\left(E_{K} \otimes P_{\xi}\right) v & =v^{T}\left(E_{0} \otimes P_{\xi}\right)\left(\mathcal{J}+2 \Sigma_{\tau}\right) v-\mathbf{f}^{T}\left(E_{0} \otimes P_{\xi}\right) \Sigma_{\tau} v-v^{T}\left(E_{0} \otimes P_{\xi}\right) \Sigma_{\tau} \mathbf{f} \\
& +v^{T}\left(P_{\tau} \otimes E_{0}\right)\left(A+2 \Sigma_{L}\right) v+v^{T}\left(P_{\tau} \otimes E_{0}\right) \Sigma_{L} \mathbf{g}+\mathbf{g}^{T}\left(P_{\tau} \otimes E_{0}\right) \Sigma_{L} v \\
& +v^{T}\left(P_{\tau} \otimes E_{N}\right)\left(-A+2 \Sigma_{R}\right) v+v^{T}\left(P_{\tau} \otimes E_{N}\right) \Sigma_{R} \mathbf{h}+\mathbf{h}^{T}\left(P_{\tau} \otimes E_{N}\right) \Sigma_{R} v \\
& +v^{T} \mathcal{P}\left(\mathcal{J}_{\tau}+A_{\xi}\right) v
\end{aligned}
$$


where $E_{K}=\operatorname{diag}(0, \ldots, 1)$.

The last term on the right hand side of (4.14), by using (4.13), becomes

$$
\mathcal{J}_{\tau}+A_{\xi}=\operatorname{diag}\left[\mathcal{D}_{\tau}\left(\mathcal{D}_{\xi} X\right)-\mathcal{D}_{\xi}\left(\mathcal{D}_{\tau} X\right)\right]=\operatorname{diag}\left[\left(D_{\tau} \otimes D_{\xi}-D_{\tau} \otimes D_{\xi}\right) X\right]=\mathbf{0},
$$

where $\mathbf{0}$ denotes the zero matrix.

Remark 4. The relation in (4.15) is the numerical version of (4.5), referred to as the Numerical GCL (NGCL). If one uses another type of discretization in time, such that time and space discretizations do not commute, (4.15) will not necessarily hold. This may result in an instability [23].

The scheme (4.14) is stable by the choices

$$
\begin{array}{rlrl}
\Sigma_{\tau} & \leq-\mathcal{J} / 2, & & \\
\left(\Sigma_{L}\right)_{j j} \leq-\left(A_{L}\right)_{j j} / 2 & \text { if } \quad\left(A_{L}\right)_{j j}>0, \\
\left(\Sigma_{L}\right)_{j j}=0 & \text { if } \quad\left(A_{L}\right)_{j j} \leq 0, \\
\left(\Sigma_{R}\right)_{j j} \leq\left(A_{R}\right)_{j j} / 2 & \text { if } \quad\left(A_{R}\right)_{j j}<0, \\
\left(\Sigma_{R}\right)_{j j}=0 & \text { if } \quad\left(A_{R}\right)_{j j} \geq 0,
\end{array}
$$

where $j \in\{0,1, \ldots,(K+1) \times(N+1)\}$. In (4.16), $A_{L}$ and $A_{R}$ indicate restrictions to the left and right boundary locations. We make the specific choice

$$
\Sigma_{\tau}=-\mathcal{J}, \Sigma_{L}=-\left(A_{L}+\left|A_{L}\right|\right) / 2, \Sigma_{R}=\left(A_{R}-\left|A_{R}\right|\right) / 2,
$$

which satisfy (4.16).

By inserting (4.17) into (4.14), the result can be written in a compact form as

$$
\begin{aligned}
\left\|v_{K}\right\|_{H_{K}}^{2} & +v_{L}^{T}\left|A_{L}^{-}\right|\left(P_{\tau} \otimes E_{0}\right) v_{L}+v_{R}^{T} A_{R}^{+}\left(P_{\tau} \otimes E_{N}\right) v_{R}=\left\|\mathbf{f}||_{H_{0}}^{2}-\right\| v_{0}-\mathbf{f} \|_{H_{0}}^{2} \\
& +\mathbf{g}_{L}^{T} A_{L}^{+}\left(P_{\tau} \otimes E_{0}\right) \mathbf{g}_{L}-\left(v_{L}-\mathbf{g}_{L}\right)^{T} A_{R}^{+}\left(P_{\tau} \otimes E_{0}\right)\left(v_{L}-\mathbf{g}_{L}\right) \\
& +\mathbf{h}^{T}\left|A_{R}^{-}\right|\left(P_{\tau} \otimes E_{N}\right) \mathbf{h}-\left(v_{R}-\mathbf{h}\right)^{T}\left|A_{R}^{-}\right|\left(v_{R}-\mathbf{h}\right) .
\end{aligned}
$$

In (4.18), subscripts $0, K, L, R$ restrict the solution to the initial time, final time, left and right boundaries, respectively. The superscripts + and - denote restrictions to positive and negative elements. Additionally, $\left\|v_{K, 0}\right\|_{H_{K, 0}}^{2}=v_{K, 0}^{T} H_{0, K} v_{K, 0}$, where $H_{K, 0}=$ $\mathcal{J}\left(E_{K, 0} \otimes P_{\xi}\right)$ are positive definite when acting on the solutions $v_{K, 0}$. The estimate in (4.18) is similar to its continuous counterpart (4.10).

Remark 5. The techniques discussed in (4.1) and (4.2) are used in papers I-V but for multiple dimensions.

\subsection{The interface procedure}

In this section, the fully discrete SBP-SAT technique for deforming interface procedure is described and compared with the fixed domain results in Section 3.3. 

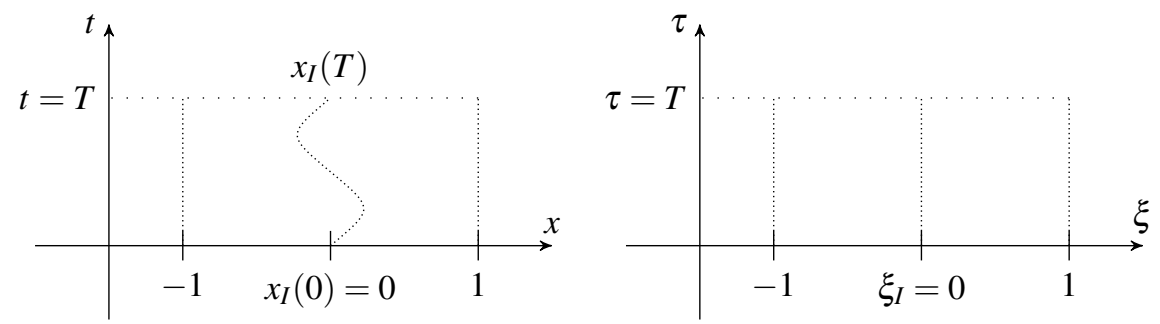

Figure 4.2: A schematic of the left and right domains, and the deforming interface.

\subsubsection{The continuous case}

Consider the constant coefficient advection equation with the solution $u$ posed on two nearby deforming spatial domains as

$$
\begin{aligned}
& u_{t}^{L}=-a u_{x}^{L}, \quad x \in\left[-1, x_{I}(t)\right] \\
& u_{t}^{R}=-a u_{x}^{L}, \quad x \in\left[x_{I}(t), 1\right],
\end{aligned}
$$

where $a>0, u^{L, R}$ denote the left and right values of the solution and $x_{I}(t)$ represents the position of the interface.

Two time-dependent Lagrangian-Eulerian coordinate transformations of the form (4.2) map the deforming domains into fixed ones with a time-independent interface, $\xi_{I}$, in between. The transformations are in general different, and will therefore be distinguished by the superscripts. Both transformations satisfy (4.3), (4.4) and (4.5). A schematic of the deforming and fixed domains is given in Figure 4.2. The transformations take (4.19) to

$$
\begin{aligned}
& J^{L} u_{\tau}^{L}=-\alpha^{L} u_{\xi}^{L}, \quad \xi \in[-1,0] \\
& J^{R} u_{\tau}^{R}=-\alpha^{R} u_{\xi}^{L}, \quad \xi \in[0,1],
\end{aligned}
$$

where $\alpha^{L, R}=\left(J \xi_{t}\right)^{L, R}+a\left(J \xi_{x}\right)^{L, R}$.

\section{Conservation}

The conservation is studied by multiplying (4.20) with a smooth function $\phi(\tau, \xi)$ with compact support at the spatial and temporal boundaries. Integrating in time and space together with the use of the GCL yield

$$
\begin{aligned}
\int_{0}^{T} \int_{-1}^{0} u^{L}\left[\left(J^{L} \phi\right)_{\tau}+\left(\alpha^{L} \phi\right) \xi\right] d \xi d \tau & +\int_{0}^{T} \int_{0}^{1} u^{R}\left[\left(J^{R} \phi\right)_{\tau}+\left(\alpha^{R} \phi\right) \xi\right] d \xi d \tau \\
& =\int_{0}^{T} \phi\left(\alpha_{I}^{L} u_{I}^{L}-\alpha_{I}^{R} u_{I}^{R}\right) d \tau
\end{aligned}
$$

Due to the continuity at the interface, $u^{L}(\tau, 0)=u^{R}(\tau, 0)$, the requirement

$$
\alpha^{L}(\tau, 0)=\alpha^{R}(\tau, 0)
$$


removes the interface term on the right hand side of (4.21) and leads to a conservative formulation. Hence, to avoid non-physical growth, the left and right metric terms must have identical values at the interface. This requirement must be respected also in the discrete case as we will show later.

Remark 6. The requirement (4.22) is due to the deformation of the interface. Such a requirement was not necessary in Section (3.3.1), because the wave speeds in (3.14) are equal by construction.

\section{Well-posedness}

Applying the energy method to (4.20) give

$$
\begin{aligned}
\left\|u^{L}(T, x)\right\|_{J^{L}}^{2}+\left\|u^{R}(T, x)\right\|_{J^{R}}^{2}= & -\int_{0}^{T} \alpha^{L}\left(u^{L}(\tau, 0)\right)^{2} d \tau+\int_{0}^{T} \alpha^{R}\left(u^{R}(\tau, 0)\right)^{2} d \tau \\
& +\left\|u^{L}(0, x)\right\|_{J^{L}}^{2}+\left\|u^{R}(0, x)\right\|_{J^{R}}^{2} .
\end{aligned}
$$

Continuity at the interface, the requirement (4.22) and the initial conditions $u^{L}=f^{L}$ and $u^{R}=f^{R}$, take (4.23) to

$$
\left\|u^{L}(T, \xi)\right\|_{J^{L}}^{2}+\left\|u^{R}(T, \xi)\right\|_{J^{R}}^{2}=\left\|f^{L}\right\|_{J^{L}}^{2}+\left\|f^{R}\right\|_{J^{R}}^{2} .
$$

The estimate (4.24) shows that the solutions have no artificial growth due to the interface.

\subsubsection{The discrete case}

We discretize the left and right spatial domains by using uniform grids of size $N+1$ and $M+1$, respectively, and introduce $K+1$ equidistant time steps. The fully discrete left and right numerical solutions, denoted by $v^{L}$ and $v^{R}$, are arranged in a similar way to $(3.6)$.

The SBP-SAT version of (4.20) (after using the splitting technique [19]), including only the initial and interface treatments, is

$$
\begin{aligned}
\frac{1}{2}\left[\partial^{L} \mathcal{D}_{\tau}^{L} v^{L}+\mathcal{D}_{\tau}^{L} \partial^{L} v^{L}+\partial_{\tau}^{L} v^{L}\right]= & -\frac{1}{2}\left[A^{L} \mathcal{D}_{\xi}^{L} v^{L}+\mathcal{D}_{\xi}^{L} A^{L} v^{L}+A_{\xi}^{L} v^{L}\right] \\
& +\Sigma^{L}\left[I_{\tau} \otimes\left(P_{\xi}^{L}\right)^{-1} E_{N}\right]\left(v^{L}-\mathbf{v}^{R}\right) \\
& +\Sigma_{\tau}^{L}\left(P_{\tau}^{-1} E_{0} \otimes I_{\xi}^{L}\right)\left(v^{L}-\mathbf{f}^{L}\right), \\
\frac{1}{2}\left[\partial^{R} \mathcal{D}_{\tau}^{R} v^{R}+\mathcal{D}_{\tau}^{R} \partial^{R} v^{R}+\partial_{\tau}^{R} v^{R}\right]= & -\frac{1}{2}\left[A^{R} \mathcal{D}_{\xi}^{R} v^{R}+\mathcal{D}_{\xi} A^{R} v^{R}+A_{\xi}^{R} v^{R}\right] \\
& +\Sigma^{R}\left[I_{\tau} \otimes\left(P_{\xi}^{R}\right)^{-1} E_{0}\right]\left(v^{R}-\mathbf{v}^{L}\right) \\
& +\sum_{\tau}^{R}\left(P_{\tau}^{-1} E_{0} \otimes I_{\xi}^{R}\right)\left(v^{R}-\mathbf{f}^{R}\right),
\end{aligned}
$$

where $\mathcal{J}^{L, R}, \partial_{\tau}^{L, R}, A^{L, R}$ and $A_{\xi}^{L, R}$ are constructed as in (4.13). By $\mathbf{v}_{I}^{R}$ and $\mathbf{v}_{I}^{L}$, we denote vectors with the size of the left and right schemes, respectively, having the right and left interface solutions at relevant positions. Additionally, $\Sigma^{L, R}$ are diagonal penalty operators for the weak imposition of interface conditions for the left and right schemes, respectively. Moreover, $\Sigma_{\tau}^{L, R}$ is a diagonal penalty operator for the weak imposition of the left and right initial conditions. Finally, $\mathbf{f}^{L, R}$ are zero vectors with initial data $f^{L, R}$ injected into positions corresponding to the initial time. 


\section{Discrete conservation}

The discrete conservation is studied by multiplying (4.25) with $\phi^{T} \mathcal{P}^{L, R}$ where $\mathcal{P}^{L, R}=P_{\tau} \otimes P_{\xi}^{L, R}$ together with the use of the $N G C L$. The result is

$$
\begin{array}{r}
\frac{1}{2}\left[\left(v^{L}\right)^{T} \mathcal{P}^{L}\left(\partial^{L} \mathcal{D}_{\tau}^{L}+\mathcal{D}_{\tau}^{L} \partial^{L}+\partial_{\tau}^{L}+A^{L} \mathcal{D}_{\xi}^{L}+\mathcal{D}_{\xi}^{L} A^{L}+A_{\xi}^{L}\right) \phi\right]^{T}+ \\
\frac{1}{2}\left[\left(v^{R}\right)^{T} \mathcal{P}^{R}\left(\partial^{R} \mathcal{D}_{\tau}^{R}+\mathcal{D}_{\tau}^{R} \partial^{R}+\partial_{\tau}^{R}+A^{R} \mathcal{D}_{\xi}^{R}+\mathcal{D}_{\xi}^{R} A^{R}+A_{\xi}^{R}\right) \phi\right]^{T}= \\
\phi_{I}^{T} P_{\tau}\left[A_{I}^{L} v_{I}^{L}-A_{I}^{R} v_{I}^{R}+\left(\Sigma_{I}^{R}-\Sigma_{I}^{L}\right)\left(v_{I}^{R}-v_{I}^{L}\right)\right] .
\end{array}
$$

where the subscript $I$ indicates interface. The matrices and vectors on the right hand side of (4.26) are of size $K+1$.

As mentioned before, the matrices $A^{L}$ and $A^{R}$ include pointwise approximations to $\alpha^{L}$ and $\alpha^{R}$, respectively, using (4.13). At the interface, we have $A_{I}^{L}=A_{I}^{R}:=A_{I}$, which corresponds to the continuous requirement in (4.22). The right hand side of (4.26) becomes

$$
\phi_{I}^{T} P_{\tau}\left(A_{I}-\Sigma_{I}^{L}+\Sigma_{I}^{R}\right)\left(v_{I}^{L}-v_{I}^{R}\right) .
$$

In order to obtain a conservative scheme, $\Sigma_{I}^{L, R}$ must be chosen such that

$$
A_{I}-\Sigma_{I}^{L}+\Sigma_{I}^{R}=0
$$

holds, by which the right hand side of (4.26) vanishes. Note the similarity of (4.28) to (3.20).

\section{Stability}

The discrete energy method and the choices $\Sigma_{\tau}^{L, R}=-\mathcal{J}^{L, R}$ take (4.25) to

$$
\begin{aligned}
\left\|v_{K}^{L}\right\|_{H_{K}^{L}}^{2}+\left\|v_{K}^{R}\right\|_{H_{K}^{R}}^{2} & =\left[\begin{array}{c}
v_{I}^{L} \\
v_{I}^{R}
\end{array}\right]^{T}\left[\begin{array}{cc}
P_{\tau} & 0 \\
0 & P_{\tau}
\end{array}\right]\left[\begin{array}{cc}
2 \Sigma_{I}^{L}-A_{I} & -\left(\Sigma_{I}^{L}+\Sigma_{I}^{R}\right) \\
-\left(\Sigma_{I}^{L}+\Sigma_{I}^{R}\right) & 2 \Sigma_{I}^{R}+A_{I}
\end{array}\right]\left[\begin{array}{c}
v_{I}^{L} \\
v_{I}^{R}
\end{array}\right] \\
& +\left\|\mathbf{f}^{L}\right\|_{H_{0}^{L}}^{2}-\left\|v_{0}^{L}-\mathbf{f}^{L}\right\|_{H_{0}^{L}}^{2}+\left\|\mathbf{f}^{R}\right\|_{H_{0}^{R}}^{2}-\left\|v_{0}^{R}-\mathbf{f}^{R}\right\|_{H_{0}^{R}}^{2} .
\end{aligned}
$$

where the subscripts $K$ and 0 denote restrictions to $\tau_{K}=T$ and $\tau_{0}=0$. Additionally, $H_{K}^{L, R}=\mathcal{J}^{L, R}\left(E_{K} \otimes p_{\xi}^{L, R}\right)$ and $H_{0}^{L, R}=\mathcal{J}^{L, R}\left(E_{0} \otimes P_{\xi}^{L, R}\right)$.

By considering the conservation requirement (4.28), (4.29) becomes

$$
\begin{aligned}
\left\|v_{K}^{L}\right\|_{H_{K}^{L}}^{2}+\left\|v_{K}^{R}\right\|_{H_{K}^{R}}^{2} & =\left(v_{I}^{L}-v_{I}^{R}\right)^{T} P_{\tau}\left(-A_{I}+2 \Sigma_{I}^{L}\right)\left(v_{I}^{L}-v_{I}^{R}\right) \\
& +\left\|\mathbf{f}^{L}\right\|_{H_{0}^{L}}^{2}-\left\|v_{0}^{L}-\mathbf{f}^{L}\right\|_{H_{0}^{L}}^{2}+\left\|\mathbf{f}^{R}\right\|_{H_{0}^{R}}^{2}-\left\|v_{0}^{R}-\mathbf{f}^{R}\right\|_{H_{0}^{R}}^{2} .
\end{aligned}
$$

From (4.30), we conclude that the following condition leads to a stable and conservative interface treatment

$$
\Sigma_{I}^{L} \leq A_{I} / 2 .
$$

Note the similarity of (4.31) to (3.23). 
We choose $\Sigma_{I}^{L}=\left(A_{I}-\left|A_{I}\right|\right) / 2$ and $\Sigma_{I}^{R}=-\left(A_{I}+\left|A_{I}\right|\right) / 2$ by which (4.28) and (4.31) are satisfied and (4.30) becomes

$$
\begin{aligned}
\left\|v_{K}^{L}\right\|_{H_{K}^{L}}^{2}+\left\|v_{K}^{R}\right\|_{H_{K}^{R}}^{2}= & -\left(v_{I}^{L}-v_{I}^{R}\right)^{T} P_{\tau}\left|A_{I}\right|\left(v_{I}^{L}-v_{I}^{R}\right) \\
& +\left\|\mathbf{f}^{L}\right\|_{H_{0}^{L}}^{2}-\left\|v_{0}^{L}-\mathbf{F}^{L}\right\|_{H_{0}^{L}}^{2}+\left\|\mathbf{f}^{R}\right\|_{H_{0}^{R}}^{2}-\left\|v_{0}^{R}-\mathbf{f}^{R}\right\|_{H_{0}^{R}}^{2} .
\end{aligned}
$$

The estimate in (4.32) approximates (4.24), and includes additional damping terms. It is also similar to the estimate (3.24) for fixed domain.

Remark 7. The conservation study in this chapter is used in paper III, for hyperbolic systems posed on deforming geometries in several space dimensions. 


\section{Bibliography}

[1] H.-O. Kreiss and J. Oliger, Comparison of accurate methods for the integration of hyperbolic equations, Tellus 24(3):199-215 (1972).

[2] H.-O. Kreiss and G. Scherer, Finite element and finite difference methods for hyperbolic partial differential equations, Mathematical Aspects of Finite Elements in Partial Differential Equations, 33:195-212 (1974).

[3] B. Strand, Summation by parts for finite difference approximations of d/dx, Journal of Computational Physics, 110(1):47-67 (1994).

[4] J. Hadamard, Lectures on Cauchy's problem in linear partial differential equations, Dover Phoenix, New York, NY, USA (1923).

[5] H.-O. Kreiss, Initial boundary value problems for hyperbolic systems, Communications on Pure and Applied Mathematics, 23(3):277-298 (1970).

[6] K. Mattsson and J. Nordström, Summation by parts operators for finite difference approximations of second derivatives, Journal of Computational Physics, 199(2):503-540 (2004).

[7] M. H. Carpenter and D. Gottlieb and S. Abrabanel, Time-stable boundary conditions for finite-difference schemes solving hyperbolic systems: methodology and application to high-order compact schemes, Journal of Computational Physics, 111(2):220-236 (1994).

[8] J. Nordström and M. H. Carpenter, High-order finite difference methods, multidimensional linear problems and curvilinear coordinates, Journal of Computational Physics, 173:149-174 (2001). 
[9] M. Svärd and M. H. Carpenter and J. Nordström, A stable high-order finite difference scheme for the compressible Navier-Stokes equations: far-field boundary conditions, Journal of Computational Physics, 225(1):1020-1038 (2007).

[10] J. E. Kozdon and E. M. Dunham and J. Nordström, Simulation of dynamic earthquake ruptures in complex geometries using high-order finite difference methods, Journal of Scientific Computing, 55(1):92-124 (2013).

[11] X. Deng and M. Mao and G. Tu and H. Zhang and Y. Zhang, High-order and high accurate CFD methods and their applications for complex grid problems, Journal of Computational and Applied Mathematics, 11(4):1081-1102 (2012).

[12] J. Nordström and R. Gustafsson, High order finite difference approximations of electromagnetic wave propagation close to material discontinuities, Journal of Scientific Computing, 18(2):215-234 (2003).

[13] J. Nordström and J. Gong and J. E. van der Weide and M. Svärd, A stable and conservative high order multi-block method for the compressible Navier-Stokes equations, Journal of Computational Physics, 228(24):9020-9035 (2009).

[14] J. Nordström and M. H. Carpenter, Boundary and interface conditions for high order finite difference methods applied to the Euler and Navier Stokes equations, Journal of Computational Physics, 148(2):621-645 (1999).

[15] M. Svärd and J. Nordström, Review of summation-by-parts schemes for initialboundary-value problems, Journal of Computational Physics, 268: 17-38 (2014).

[16] D. C. Del Rey Fernández and J. E. Hicken and D. W. Zingg, Review of summationby-parts operators with simultaneous approximation terms for the numerical solution of partial differential equations, Computers \& Fluids, 95: 171-196 (2014).

[17] B. Gustafsson, The convergence rate for difference approximations to general mixed initial-boundary value problems, SIAM Journal of Numerical Analysis, 18(2):179-190 (1981).

[18] B. Gustafsson, The convergence rate for difference approximations to mixed initial boundary value problems, Mathematics of Computations, 29(130):396-406 (1975).

[19] J. Nordström, Conservative finite difference formulations, variable coefficients, energy estimates and artificial dissipation, Journal of Scientific Computing, 29(3):375-404 (2006).

[20] J. Nordström and T. Lundquist, Summation-by-parts in time, Journal of Computational Physics, 251:487-499 (2013).

[21] T. Lundquist and J. Nordström, The SBP-SAT technique for initial value problems, Journal of Computational Physics, 270:86-104 (2014).

[22] J. Nordström and T. Lundquist, Summation-by-parts in time: the second derivative, SIAM Journal of Scientific Computing, 38(3):A1561-A1586 (2016).

[23] H. Guillard and C. Farhat, On the significance of the geometric conservation law for flow computations on moving meshes, Computer Methods in Applied Mechanics and Engineering, 190(11):1467-1482 (2000). 


\section{5}

\section{Summary of papers}

Paper I - Fully discrete energy stable high order finite difference methods for hyperbolic problems in deforming domains

We consider hyperbolic IBVPs posed on time-dependent deforming domains. To incorporate the movements of the physical geometry, a time-dependent coordinate transformation is employed to map the governing equations to a fixed domain. The transformation results in variable coefficient problems and poses challenges such as satisfying the NGCL and the need for a time-dependent penalty formulation.

Prior to constructing the scheme, well-posedness and conservation are studied by using the energy method. Next, we use a special splitting technique required for problems with variable coefficients and construct the SBP-SAT scheme. Stability and conservation are proved by employing the discrete energy method. Moreover, we show how to construct the scheme such that the NGCL is satisfied. Details on how to construct the SAT penalty operators such that the scheme automatically adjusts to the movements and deformations of the geometry are also provided.

In the numerical experiments, calculations for the linearized Euler equations posed on time-dependent moving domains are presented. We show that the scheme is design order accurate and error-bounded. Additionally, it is shown that the free-stream property is preserved after long time calculations. An application in sound propagation governed by the Euler equations is also provided, showing that the behavior of the approximated quantities such as the pressure distribution and the velocity field is correct.

Paper II - Hyperbolic systems of equations posed on erroneous curved domains

In many real world applications, geometries are not exactly described. The erroneous geometries are typically caused by faulty mesh generators, CAD systems, round off or measurement errors. In this article, the effects of inaccurate geometries on the accuracy 
of the solution are studied.

The theoretical analysis is done by considering two geometries, one exact and one erroneous, in a curvilinear coordinate system. Both geometries are then mapped to a reference domain. The effects of the erroneous representation of the geometry, are studied by looking closer at the metric coefficients. Different scenarios that relate the accuracy of the solution to the erroneously described geometry are addressed. Lastly, numerical experiments that support the theoretical predictions are shown.

The conclusions are: the erroneous spatial domain may lead to erroneous wave speeds, a misplacement of the boundary condition, to erroneous boundary operators and a mismatch of boundary data. In many cases, these errors will affect the global accuracy of the solution more than the order of accuracy.

Paper III - A fully discrete, stable and conservative summation-by-parts formulation for deforming interfaces

In many applications, one has to divide the spatial geometry into different parts and couple the problems at the interfaces. In a more complicated situation, the domains and the interfaces between them are, in a time-dependent manner, deforming. In this paper, the SBP-SAT procedure for moving domains given in paper $I$ is extended to moving and deforming interfaces.

In the theoretical part, the continuous and discrete energy methods are the main analytical tools. Well-posedness, continuous and discrete conservation as well as stability are studied. Numerical calculations using the linearized Euler equations and the Maxwell equations are presented which confirm the stability and accuracy of the schemes.

Paper IV - A dual consistent summation-by-parts formulation for the linearized incompressible Navier-Stokes equations posed on deforming domains

The linearized incompressible Navier-Stokes equations posed on a two dimensional deforming domain is considered. The problem is reduced into a first order system to make the derivations of the dual problem easier. Next, we transform the problem to a fixed domain using a time-dependent coordinate transformation. Lastly, the dual problem is derived and well-posedness of the primal and dual problems is studied.

The discrete primal and dual problems are constructed on SBP-SAT form where the NGCL is satisfied. Stability of both schemes are investigated by using the discrete energy method. The two approximations are made dual consistent by choosing specific penalty operators among those that are appropriate for stability.

In the numerical part, we show that the convergence rates of the solutions obtained are correct. Additionally, the divergence of the solution converges with the design rate of the scheme, due to the formulation as a first order system. Moreover, we show that linear and nonlinear functionals of the solution superconverge.

\section{Paper V - Summation-by-parts operators for non-simply connected domains}

In this paper, we apply the SBP-SAT technique to problems posed on non-simply connected spatial domains. The problem considered in the theoretical part is the two 
dimensional constant coefficient advection equation posed on a rectangular domain with a hole. We study the stability and show that the eigenvalues of the semi-discrete problem have the correct sign, which means that stability follows together with the SBP-SAT technique in time.

In the numerical experiments, we consider the linearized Euler equations posed on a rectangular geometry with a hole, where we show the convergence rates for schemes of different orders of accuracy. Additionally, we make a comparison between the new approach and the standard interface procedure in terms of accuracy and efficiency. Finally, we present an application using the linearized Euler equations on a rectangular geometry with several holes. 



\section{Papers}





\section{Papers}

The articles associated with this thesis have been removed for copyright reasons. For more details about these see:

http://urn.kb.se/resolve?urn=urn:nbn:se:liu:diva-130928 